\title{
Complex organic molecules in the intermediate mass protostar OMC2-FIR4
}

\author{
Nicolas Crimier, Cecilia Ceccarelli, Bertrand Lefloch, and Alex Faure \\ Laboratoire d'Astrophysique de Grenoble, Université Joseph Fourier, UMR 5571-CNRS, \\ France \\ email: ncrimier@obs.ujf-grenoble.fr
}

\begin{abstract}
We present the physical structure (density and temperature profiles) and the distribution of formaldehyde and methanol in intermediate mass protostar OMC2-FIR4 in the Orion molecular cloud complex.
\end{abstract}

Keywords. Astrochemistry, ISM: individual (OMC2-FIR4), line: formation

\section{Introduction}

The first stages of star evolution are characterized, among other things, by the formation Complex Organic Molecules (COMs; see e.g. Ceccarelli's contribution in this volume). Before the collapse sets in, in the pre-stellar condensations, at least some simple COMs like formaldehyde and methanol are synthesized on the grain surfaces by hydrogenation of CO. The birth of a protostar at the center warms up the surrounding matter up to $100 \mathrm{~K}$ or more, with two consequences: the previously formed grain ice mantles sublimate injecting into the gas phase their molecular content, and, second, new and more complex molecules are synthesized in the gas phase because of new formation routes opening up at the large gas temperatures and the availability of the molecules synthesized on the grain surfaces. What and how much COMs are formed at the end depend on the mass of the protostar, as suggested by some comparative studies between low and high mass protostars (Bottinelli et al. 2007). Surprising enough, low mass protostars synthesize larger quantities of COMs with respect to high mass protostars. This may be the result of both the COMs formation and destruction processes. Here we present new observations of methanol and formaldehyde, the simplest of but the most crucial COMs, towards the intermediate mass (IM) protostar OMC2-FIR4, the brightest submillimeter source of the Orion Molecular Cloud 2 with a bolometric luminosity of $1000 \mathrm{~L}_{\odot}$.

\section{Density, temperature, and $\mathrm{CH}_{3} \mathrm{OH}$ and $\mathrm{H}_{2} \mathrm{CO}$ abundance profiles}

We derived the density and dust temperature profiles of the protostar OMC2-FIR4 envelope, by modeling the dust continuum emission by means of the radiative transfer code DUSTY (Ivezic \& Elitzur 1999). We considered the Spectral Energy Density (SED) between $1.3 \mathrm{~mm}$ and $24 \mu \mathrm{m}$ and the maps at 350, 450 and $850 \mu \mathrm{m}$ respectively (see Crimier et al. 2008 for details). We assumed a power low density profile, $n(r)=n_{0}\left(r / r_{0}\right)^{-\alpha}$, for an envelope of radius $R_{\max } \cdot n_{0}, \alpha$ and $R_{\max }$ are then constrained by comparison between the observations and the model predictions. Following the claim by Jørgensen et al. 2006 that an Inter-Stellar Radiation Field (ISRF) with $G_{0}=10^{4}$ illuminates the OMC2-FIR4 envelope, we run models with $G_{0}$ between 1 and $10^{4}$. We found that the continuum observations cannot constrain the $G_{0}$ value: all the ran models yield similar $\chi^{2}$ values. 
Deriving correct abundances from observations of molecular lines requires knowledge of the gas temperature profile. Very often, the approximation of $T_{\text {gas }}=T_{\text {dust }}$ is assumed. While this is a good enough approximation in low mass protostars, in IM mass protostars it may break down. Thus we modeled the thermal balance across the envelope, following the method described in Ceccarelli et al. (1996). To test the hypothesis of large $G_{0}$ illuminating OMC2-FIR4 we run 2 models with $G_{0}=1$ and 1000 respectively. Moreover, a crucial parameter in the thermal coupling between dust and gas is the water abundance, as water is a major coolant of the gas. Figure 1 presents the gas temperature and density (found by the modeling of the continuum discussed in $\S 2$ ) profiles in the two cases $G_{0}=1$ and 1000, and for different $\mathrm{H}_{2} \mathrm{O}$ abundances in the inner part. The dust and gas are thermally decoupled in the inner region $\left(\mathrm{T}_{\text {dust }} \geqslant 100 \mathrm{~K}\right)$, because of the water ices sublimation.
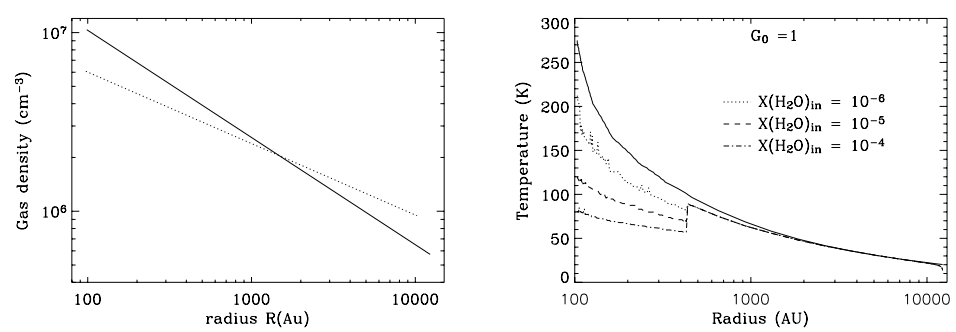

Figure 1. The gas density profile (left panel) for $\mathrm{G}_{0}$ equal to 1 (plain curve) and 1000 (pointed curve) and temperature profile (right panel) for different values of the water abundance $\mathrm{X}\left(\mathrm{H}_{2} \mathrm{O}\right)_{i n}$.

We obtained multi-frequency observations of o- $\mathrm{H}_{2} \mathrm{CO}, \mathrm{p}-\mathrm{H}_{2} \mathrm{CO}$ and $\mathrm{CH}_{3} \mathrm{OH}$ at the IRAM and JCMT telescopes (details in Crimier et al., in prep.). Following the method by Maret et al. (2004) we derived the $\mathrm{o}-\mathrm{H}_{2} \mathrm{CO}, \mathrm{p}-\mathrm{H}_{2} \mathrm{CO}$ and $\mathrm{CH}_{3} \mathrm{OH}$ abundances in the inner and outer envelope of OMC2-FIR4 (Table 1). For a first analysis, we assumed LTE level distribution. From this preliminary analysis, the $\chi\left(\mathrm{H}_{2} \mathrm{CO}\right) / \chi\left(\mathrm{CH}_{3} \mathrm{OH}\right)$ ratio in the inner region is $\sim 0.5$, a value in between what found in Hot Corinos $(\sim 1)$ and Hot Cores $(\sim 0.1)$ (Bottinelli et al. 2007).

Table 1. o- $\mathrm{H}_{2} \mathrm{CO}$, p- $\mathrm{H}_{2} \mathrm{CO}$ and $\mathrm{CH}_{3} \mathrm{OH}$ column densities and abundances.

\begin{tabular}{|c|c|c|c|c|c|}
\hline Molecules & $\mathrm{T}_{G A S}$ & $N_{x}\left(\mathrm{~cm}^{-2}\right)$ & $\begin{array}{l}\text { Comments } \\
\text { (opacities) }\end{array}$ & $\begin{array}{l}\text { Abundances } \\
G_{0}=1\end{array}$ & $G_{0}=10^{3}$ \\
\hline $\mathrm{CH}_{3} \mathrm{OH}$ & $\begin{array}{l}20 \mathrm{~K} \\
80 \mathrm{~K}\end{array}$ & $\begin{array}{l}\sim 2.10^{15} \\
\sim 8.10^{15}\end{array}$ & $\begin{array}{l}\text { Moderately thick } \\
\text { thin }\end{array}$ & $\begin{array}{l}\sim 6.7 .10^{-9} \\
\sim 1.5 .10^{-7}\end{array}$ & $\begin{array}{l}\sim 1.3 .10^{-8} \\
\sim 1.2 .10^{-7}\end{array}$ \\
\hline $\mathrm{p}-\mathrm{H}_{2} \mathrm{CO}$ & $\begin{array}{l}20 \mathrm{~K} \\
80 \mathrm{~K}\end{array}$ & $\begin{array}{l}\sim 5.10^{13} \\
\sim 3.10^{14}\end{array}$ & $\begin{array}{l}\text { thick } \\
\text { thin }\end{array}$ & $\begin{array}{l}\sim 1.7 .10^{-10} \\
\sim 5.5 \cdot 10^{-9}\end{array}$ & $\begin{array}{l}\sim 3.3 .10^{-10} \\
\sim 4.6 .10^{-9}\end{array}$ \\
\hline $\mathrm{o}-\mathrm{H}_{2} \mathrm{CO}$ & $\begin{array}{l}20 \mathrm{~K} \\
80 \mathrm{~K}\end{array}$ & $\begin{array}{l}\sim 1.10^{14} \\
\sim 8.10^{14}\end{array}$ & $\begin{array}{l}\text { Moderately thick } \\
\text { thin }\end{array}$ & $\begin{array}{l}\sim 3 \cdot 3 \cdot 10^{-10} \\
\sim 1 \cdot 5 \cdot 10^{-8}\end{array}$ & $\begin{array}{l}\sim 6.7 .10^{-10} \\
\sim 1.2 .10^{-8}\end{array}$ \\
\hline
\end{tabular}

\section{References}

Bottinelli, S., Ceccarelli, C., Williams, J. P., \& Lefloch, B. 2007, A\&A, 463, 601

Ceccarelli, C., Hollenbach, D. J., \& Tielens, A. G. G. M. 1996, ApJ, 471, 400

Crimier, N., Ceccarelli, C., Lefloch, B., \& Faure, A. 2008, A\&AA, submitted

Ivezic, Z. \& Elitzur, M. 1999, MNRAS, 303, 864

Jørgensen, J. K., Johnstone, D., van Dishoeck, E. F., \& Doty, S. D. 2006, A\&A, 449, 609

Maret, S., Ceccarelli, C., Caux, E. et al. 2004, A\&BA, 416, 577 